\title{
The Connection between MicroRNAs from Visceral Adipose Tissue and Non-Alcoholic Fatty Liver Disease
}

\author{
Veronika Zubáňová1,*, Zuzana Červinková2, Otto Kučera², Vladimír Palička ${ }^{1}$
}

\begin{abstract}
Non-Alcoholic Fatty Liver Disease (NAFLD) is one of the most important causes of liver disease worldwide leading the foreground cause of liver transplantation. Recently miRNAs, small non-coding molecules were identified as an important player in the negative translational regulation of many protein-coding genes involved in hepatic metabolism. Visceral adipose tissue was found to take part in lipid and glucose metabolism and to release many inflammatory mediators that may contribute to progression of NAFLD from simple steatosis to Non-Alcoholic SteatoHepatitis. Since visceral adipose tissue enlargement and dysregulated levels of miRNAs were observed in patients with NAFLD, the aim of this paper is to reflect the current knowledge of the role of miRNAs released from visceral adipose tissue and NAFLD.

\section{KEYWORDS}

non-alcoholic fatty liver disease; non-alcoholic steatohepatitis; NAFLD; NASH; microRNA; miRNA; adipose tissue; inflammation; lipid metabolism

AUTHOR AFFILIATIONS

${ }^{1}$ Department of Clinical Biochemistry and Diagnostics, Charles University, Faculty of Medicine in Hradec Králové and University Hospital Hradec Králové, Czech Republic

2 Department of Physiology, Charles University, Faculty of Medicine in Hradec Králové, Czech Republic

* Corresponding author: Department of Clinical Biochemistry and Diagnostics, Faculty Hospital Hradec Králové, Sokolská 581,50005 Hradec Králové, Czech Republic; e-mail: zubanovv@lfhk.cuni.cz
\end{abstract}

Received: 22 July 2020

Accepted: 27 November 2020

Published online: 14 April 2021

Acta Medica (Hradec Králové) 2021; 64(1): 1-7

https://doi.org/10.14712/18059694.2021.1

(c) 2021 The Authors. This is an open-access article distributed under the terms of the Creative Commons Attribution License (http://creativecommons.org/licenses/by/4.0), which permits unrestricted use, distribution, and reproduction in any medium, provided the original author and source are credited. 


\section{INTRODUCTION}

Non-Alcoholic Fatty Liver Disease (NAFLD) also known as Metabolic Associated Fatty Liver Disease (MAFLD) (1) is the most common chronic liver disease, which afflicts approximately a quarter of the worldwide population $(2,3)$. However, there also exist some variances in prevalence according to interindividual differences, such as ethnicity and gender. The highest prevalence has been reported in industrialized countries (Western Europe, USA and Middle East) and the lowest number of affected people is in Africa $(3,4)$. NAFLD is defined by liver fat accumulation in the absence of any other known cause of hepatic steatosis (extensive alcohol intake, chronic viral hepatitis, drug intake or genetic disorders etc.) (5). After hepatitis C, NAFLD is the second leading cause for liver transplantation in developed countries such as the USA. This is the result of asymptomatic follow-up in most of NAFLD patients, which may lead to late detection of the disease at an advanced stage (3).

MicroRNAs (miRNAs) are approximately 20 nucleotides long naturally occurring non-coding RNAs, which at post transcriptional level negatively regulate a large number of genes playing the role in many physiological and pathophysiological processes in the liver and in the human body in general (6-9). Until now, few thousand miRNAs were identified in human genome. miRNAs can be classified according to their characteristics and well defined role in organ development, and may be used as a very powerful, specific and sensitive biomarker in NAFLD diagnosis and follow-up as it was previously seen in many human diseases $(7,10)$. It has been suggested that microRNAs may influence almost $60 \%$ of human protein-coding genes (11). To regulate gene expression, they bind to 3 ' untranslated region (UTR) of complementary mRNA (messenger RNA) target and inhibit its translation to protein or cause mRNA degradation. According to this effect, they are involved in the regulation of many biological processes, such as glucose, cholesterol or iron homeostasis, immune response or cellular proliferation, differentiation and cell death as it was reviewed in many articles $(8,9,12-14)$. Since one miRNA can target multiple genes (miRNA multi-functionality) and multiple miRNAs may influence a single gene (redundancy), it is difficult to identify direct connection between miRNA and the target gene (9).

\section{NAFLD PATHOGENESIS AND PROGRESSION}

The pathogenesis of NAFLD is multifactorial and is triggered by environmental factors such as hypernutrition (excessive caloric intake, high intake of saturated fats, cholesterol, fructose, etc.) and physical inactivity in the context of genetic predisposition (15). Elevated incidence of NAFLD is related to the worldwide rise in metabolic syndrome. NAFLD is associated with increased risk of cardiovascular disease, metabolic syndrome and cancer development $(16,17)$. Metabolic syndrome (MS) is defined as a complex disorder including many interconnected factors (dyslipidemia, abdominal obesity, dysregulated glucose metabolism, etc.) that together contribute to higher risk of cardiovascular disease and type 2 diabetes mellitus
(18). Above $90 \%$ of patients with NAFLD have more than one component of MS and about $60 \%$ of patients with liver disease have the complete diagnosis of MS (minimal 3 of 5 signs) (19). Insulin resistance (IR) was identified as a central point in pathogenesis of NAFLD, thus considering NAFLD to be the liver manifestation of metabolic syndrome. IR leads to the retention of triacylglycerols (TAG) by the hepatocyte together with impaired carbohydrate metabolism (20). In general, insulin decreases the production of VLDL (very low-density lipoprotein) by inhibition of adipose tissue lipolysis and hepatic VLDL production. Nevertheless, in patients with NAFLD (or MS) insulin fails to inhibit lipolysis and hepatic production, leading to an increase in serum triacylglycerols and their accumulation in the liver (21). In this case, the global prevalence of NAFLD among patients with type 2 diabetes mellitus (T2DM) was estimated to be almost 60\% (22).

Several dysfunctions may participate in the pathogenesis of NAFLD such as alterations in metabolic pathways, imbalance in lipid import, de novo formation, oxidation and export, and thus leading to lipid accumulation in the liver (steatosis is defined as hepatic TAG content $>5 \%$ of liver weight) and to further progression - inflammation state (steatohepatitis) complicated with fibrosis, cirrhosis and carcinoma formation (Figure 1) (23). Non-Alcoholic SteatoHepatitis (NASH), an advanced stage of fatty liver disease, develops in $10-30 \%$ of patients with simple steatosis and in $40 \%$ of T2DM patients where is associated with a worse long-term prognosis (22). NASH is accompanied by hepatocellular damage characterized by hepatocellular ballooning, apoptosis, lobular inflammation and with or without hepatic fibrosis (23). Contrary to simple steatosis, NASH may progress to cirrhosis and, in some number of patients to hepatocellular carcinoma (23, 24). Unique miRNA profiles were found to be changed in obesity and different stages of NAFLD. Sharma et al. also found correlation of differentially expressed miRNA with various variables such as body weight, body mass index, fasting glycemia, TAG concentrations, adipocyte differentiation or adipose tissue inflammation (25). In addition to previously mentioned study, Klöting et al. found higher miR-17-5p, miR-132 and miR-134 expression and opposite pattern of miR-181a in omental fat comparing the group of patients with T2DM and normal glucose tolerance (26). Some miRNAs were identified to be dysregulated in omental and subcutaneous fat (subcutaneous adipose tissue - SAT) that significantly correlate with glucose and lipid metabolism parameters, adipose tissue morphology (macrophage infiltration, adipocyte volume) and visceral fat area, suggesting the role of miRNAs in obesity associated disorders (26). As an example, serum free fatty acid levels negatively correlate with adipose tissue level of miR-99a in human. Additionally, an expression of miR-155 was significantly related to macrophage infiltration and the strongest correlation was found between the mean omental adipocyte diameter and miR-198. These findings point out a role of miRNAs in immune cells attraction to adipose tissue and thus its proinflammatory role in NAFLD development (26). Furthermore, it has been demonstrated that visceral adipose tissue (VAT) and SAT exhibit specific gene expression patterns and in case of 
that regulate different physiological functions (27). Up to now, an exact role of miRNAs is difficult to estimate, and in silico analyses are widely used. For instance, Capobianco et al. studied miRNA and protein expression profiles of visceral adipose tissue. Based on the transcriptomic and proteomic profiles, they found a functional association between dysregulated miRNA and corresponding protein. Two pairs were identified as contributors to altered gene expression in obesity, namely, miR-141/YWHAG (tyrosine 3-monooxygenase/tryptophan 5-monooxygenase activation protein, gamma polypeptide) and miR-520e/ RAB11A (Ras-related protein). Decreased expression of miRNA was associated with up-regulation of their pairing proteins and this hypothesis was also validated by functional analysis with luciferase assay confirming binding site of these miRNAs on 3'UTR region of corresponding transcripts (28). However, further detailed research on miRNA functional studies is needed.

\section{THE ROLE OF VISCERAL ADIPOSE TISSUE IN NAFLD} Adipose tissue plays a central role in health and disease. Its primary function is to store energy in the form of triacylglycerols and release them in the usable form during fasting and starvation, when energy is needed (29). In general, obesity is characterised by an excess of white adipose tissue. Central obesity, i.e., fat accumulated in VAT, is stated to be the major risk factor for metabolic-syndrome related disorders. Although, there is considerable physiological difference in genders, whereas in men VAT represents almost $20 \%$ of total fat and in women $8 \%(16$,
29). The abdominal adipose tissue has been characterised as an endocrine tissue, releasing many hormonal signals that may affect the hypothalamic-pituitary-gonadal axis $(30,31)$. However, few epidemiological studies revealed the fact, that NAFLD occurred also in group of non-obese patients without metabolic syndrome $(32,33)$. In the group of non-obese Chinese adults, hepatic fat accumulation showed the relation to abdominal fat examined by СT (34).

\section{A) VAT inflammation}

In obesity, adipocytes enlarge their size due to higher accumulation of TAGs. However, it is hypothesised that this expansion may be limited and fat overload of adipocytes may create a hypoxic environment (35), the condition leading to adipocyte death (36). Monocytes are then attracted and infiltrate adipose tissue partially due to monocyte chemoattractant protein-1 (MCP-1), chemokine released from damaged adipocytes (37). Knockout or pharmacological blockage of MCP-1 receptor gene reduced macrophage infiltration of adipose tissue and insulin resistance in mouse on high-fat diet (38). In addition, the anatomy of the liver enables the portal and arterial circulation interaction together with hepatocytes and liver immune-system cells. In comparison to subcutaneous adipose tissue, VAT has a greater lipolytic potential and free fatty acids (FFAs) released from visceral fat are directly transported to the liver via portal circulation. These FFAs, together with exogenous fat supply and de novo lipogenesis are the main source of hepatic TAGs in NAFLD (39).

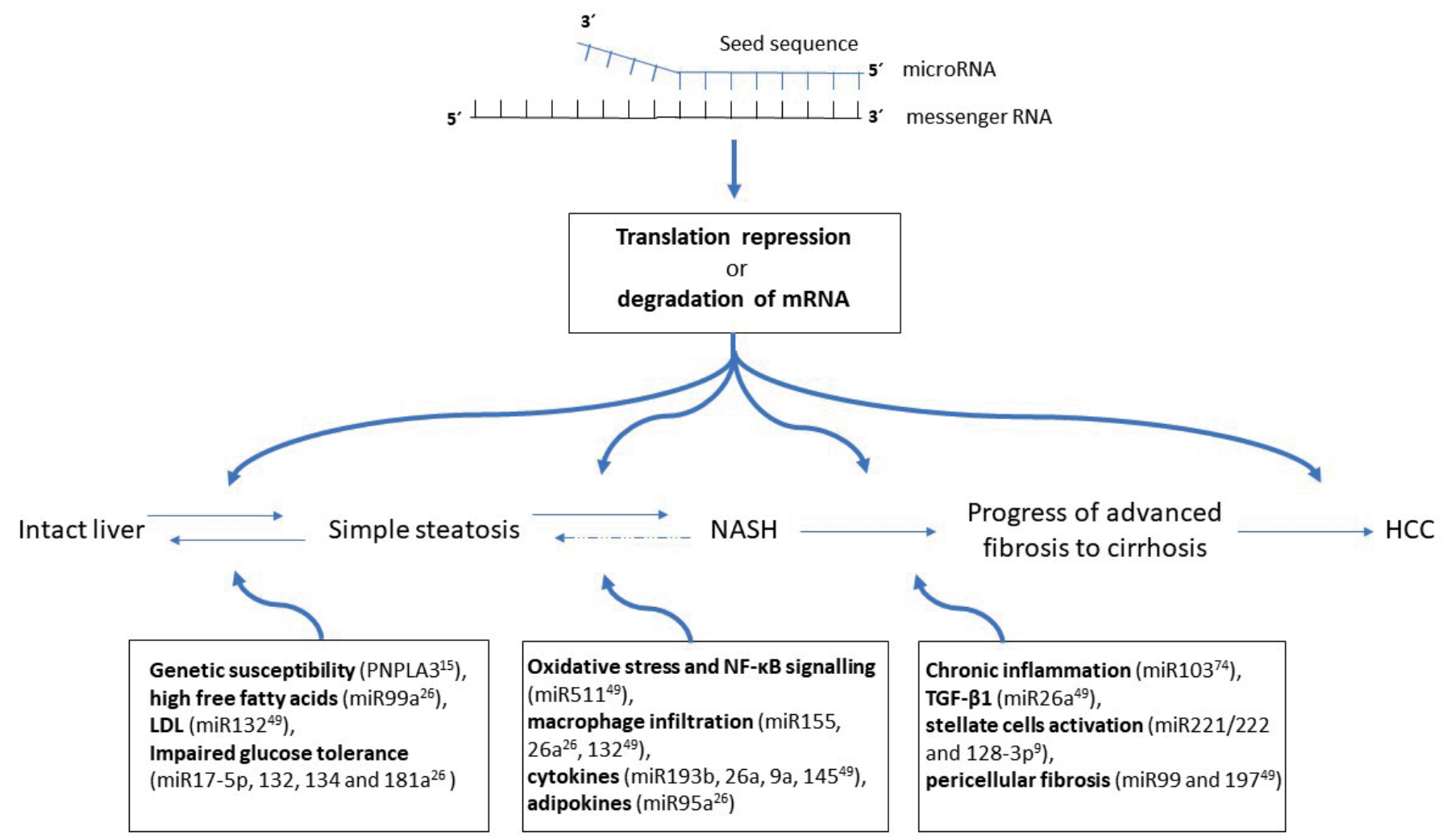

Fig. 1 Schematic diagram of possible effect of miRNAs on pathogenesis of NAFLD. HCC: Hepatocellular Carcinoma; NASH: Non-Alcoholic SteatoHepatitis; PNPLA3: Patatin-like Phospholipase Domain-containing Protein 3; TGF- $\beta 1$ : Transforming growth factor $\beta 1$. 


\section{B) VAT mediators}

The excessive expansion of VAT results in low-grade inflammation due to the presence of macrophages secreting several bioactive mediators. Dysregulation in soluble signalling molecules such as cytokines and adipokines (leptin, resistin, visfatin, interleukin 6 (IL-6) and 8 (IL-8), tumour necrosis factor alpha (TNF- $\alpha$ ), adiponectin) secreted by visceral adipose tissue can partially contribute to proinflammatory state participating on NAFLD progression to NASH, NASH-associated fibrosis and insulin resistance $(40,41)$. Since the role of adiponectin is to protect the liver from inflammation via direct antagonism of TNF- $\alpha$, dysregulation of adipocytokines may represent and important link between fat mass in obesity (metabolic syndrome) and NAFLD development (42). Adiponectin exhibits insulin-sensitising effects in the insulin-dependent tissues, such as the liver, skeletal muscle and adipose tissue. Concentrations of adiponectin were found to be downregulated in obesity and T2DM, in which inversely correlated with fat amount (43). Adipose tissue shows selective gene expression patterns, to which miRNAs contribute $(26,29,44,45)$. Enlargement of adipose tissue is associated with a transcriptional alteration of coding and also non-coding RNAs. In that case, miRNA expression from VAT may contribute to the pathogenesis of NAFLD, as it was detected in previous studies revealing their association with NAFLD $(41,46)$.

In comparison to subcutaneous adipose tissue, visceral adipose tissue contains larger adipocytes resistant to insulin and macrophages releasing pro-inflammatory cytokines such as TNF- $\alpha$, IL- 6 and C-reactive protein (47). Many miRNAs, such as miR-193b, miR-26a, miR-9a, miR-145, were found to regulate the production of MCP-1, TNF- $\alpha$ and adiponectin during visceral adipose tissue inflammatory process (48). Several adipokines have been reported to be related to changes in miRNA profile, and differential expression of miRNAs was found in the liver and also in VAT of patients with NAFLD (49). Estep et al. studied expression of miRNAs in visceral adipose tissue from NASH patients in which they found overall down-regulation of miRNA expression in comparison to non-NASH NAFLD. In addition, the decreased levels of miR-99 and miR-197 were significantly associated with pericellular fibrosis in NASH. Other miRNAs were confirmed to be associated with insulin receptor pathway (IGF1, IGFR13), cytokines (CCL3, IL6), genes connected to inflammation response (NFkB1, RELB, FAS) or ghrelin/obestatin gene. Moreover, four of differentially expressed miRNAs are positioned inside genes connected to metabolic syndrome, liver disease, obesity or T2DM, confirming their possible effect on target genes (49). This down-regulation of miRNAs was also found in the serum of NASH patients, but without any relationship to the stage of fibrosis (50). The study of Cheung et al. also supported these findings when they found eighteen miRNA species to be differentially expressed in the adipose tissue of NASH patient and confirmed their dysregulation in the liver of NASH patients (51).

In comparison, some miRNAs were found to be upregulated in adipose tissue. Chartoumpekis et al. found increased levels of miR-146a and miR-146b in obese mice fed by long-term high-fat diet (52). On the other hand, Latorre et al. investigated the decrease of few miRNAs in obese subject with NAFLD. In concordance with the results of human studies, palmitic acid used as an inductor of FA accumulation in hepatocytes in vitro, inhibited expression of many miRNAs in cell cultures, increased fatty acid transport, and fat formation, but reduces glucose metabolism and fatty acid oxidation (53). Down-regulation of miRNA miR-146a was also examined in peripheral blood mononuclear cells from T2DM patients when compared to healthy subjects suggesting the role of miR-146a in insulin resistance (54).

\section{THE ROLE OF MICRORNA IN NAFLD DIAGNOSTICS}

Until now, it has not been possible to predict NAFLD outcome through the routinely used blood and tissue markers due to their limited sensitivity and tissue specificity. The ultrasonography is a gold standard in NAFLD non-invasive diagnostics, but it has low sensitivity (detects fatty liver when approximately $30 \%$ of area is affected by liver steatosis) (55). The best tool for revealing the fine details of histopathological severity is liver biopsy. Nevertheless, this technique is invasive and could be accompanied by some severe complications, thus limiting its indication. Liver biopsy is usually performed at progressed stage of the disease and is not routinely used for mass screening or disease follow-up (56). Due to the limitations of liver biopsy, the use of non-invasive markers has emerged in recent years.

\section{A) Circulating miRnAs}

The biggest advantage of miRNAs is their use in non-invasive diagnostic due to their presence in the body fluids such as serum, plasma or urine and correlation between free circulating and tissue levels and thus reflecting the physiological/pathological state of tissue they are released from. Circulating miRNAs can result from a passive release in case of cell death or an active secretion to extracellular space in the form of microvesicles. In that case, they may indicate hepatocellular damage during liver injury or inflammation as described previously (56-58). Due to their presence in microvesicles and exosomes, they show high stability (contrary to long RNAs) in the environment containing RNases (RNA-degrading enzymes) $(56,58)$. The presence of specific surface proteins on exosomes reflects tissue or cell origin and function in cell-to-cell communications $(56,59)$. During NASH ballooning degeneration, cell death is enhanced, and many miRNAs are released into the circulation. This feature is thought to mirror molecular event in NAFLD (57). Some miRNAs also exist without cover or are incorporated in and protected by Argonaute 2 and surrounded proteins in high-density lipoproteins (HDL) $(56,58)$.

\section{RESEARCH MODELS ON THE ROLE OF MICRORNAS IN NAFLD}

Murine models play a key role in the deeper understanding of therapeutic effects and provide an opportunity for research using genetic manipulation. Popular models of obesity are genetically and diet-induced animals (mouse) 
or, on in vitro level, preadipocyte cell-culture differentiated into adipocytes (60).

Adipocyte differentiation as a cellular process is regulated by several important regulatory factors, nevertheless the whole mechanism is not fully understood. miRNAs together with insulin secretion have been shown to be involved significantly in stem cell modulation and adipocyte differentiation $(61,62)$. These results were supported by in vitro functional analysis of Esau et al. The reduction of miR-143 by transfecting antisense oligonucleotides inhibited adipocyte differentiation, suggesting that miRNAs may regulate adipose tissue processes (63). Furthermore, the study of Bergenstrate revealed almost 70 miRNAs to be differentially expressed in mesenchymal stem cell-derived adipocytes (terminally differentiated adipocytes) compared to progenitor cells. They also validated the results of in vivo studies in mouse and in vitro studies in mutant mouse line (64). Moreover, miR-21, miR-27a, miR-103a, and miR-320 have been reported to be involved in adipocyte proliferation and differentiation (65-68). The functional study demonstrated insulin-induced improvement of glucose uptake in insulin-resistant adipocytes after anti-miR-320 oligo addition. Prior to these manipulations, miR-320 expression in insulin-resistant cell line (3T3-L1) was approximately 50 times higher compared to normal cell expression. These results suggest the role of miR-320 in targeting phosphoinositide-3-kinase, regulatory subunit 1 (68). The findings are in the accordance with study of Lin et al., who demonstrated influence of miR-27 overexpression in inhibition of adipocyte formation. Also, adipogenic regulators PPAR- $\gamma$ (peroxisome proliferator-activated receptor $\gamma$ ) and $\mathrm{C} / \mathrm{EBP} \alpha$ (CCAAT-enhancer binding protein $\alpha$ ) represent targets for this miRNA, which contributes to their close link (69). PPARs are ligand-activated transcription factors from nuclear receptor subfamily that function as lipid and glucose metabolism, energetic, inflammation and atherosclerotic regulators. Few isoforms can be found in different tissues: PPAR- $\alpha$, PPAR $-\beta / \delta$ and PPAR- $\gamma$ that is highly expressed in adipose tissue (70, 71). Additionally, data from PPAR- $a$ null mice supported this hypothesis. Activation of this protein was reported to control the metabolic response of adipose tissue to diet income and induce cleavage of intracellular lipids through fatty acid oxidation (72). In adipose tissue, activation of PPAR- $\gamma$ regulates the change of the macrophages to $\mathrm{M} 2$ subtype, that exerts in contrast to M1 type, anti-inflammatory action and may be preventive in NAFLD development (73). In another study, few miRNAs were found to be pro-adipogenic (miR-143, miR-103, miR-146b, miR-148, miR-33b) and serve in the process of preadipocytes accelerated adipogenesis. This process was accompanied by increased expression of transcription factors (PPAR 2 2), cell cycle regulators, glucose transporters and adipokines (74). However, miR-143 and miR-103 were found to be significantly decreased in adipocytes from obese mice. On the contrary, miR-222 and miR-221 were downregulated during adipogenesis and conversely upregulated in adipocytes from obese subjects. In general, miRNAs that were induced during adipogenesis, were underexpressed in adipocytes from obese mice and vice versa, suggesting that these changes are related to chronic local inflammatory environment in adipose tissue (74). Intronic
miRNA - miR-33b and its target gene SREBP-1 (sterol regulatory element-binding protein 1) were also highly upregulated during preadipocyte differentiation and miR-33b inhibition decreases adipose cell differentiation and lipid droplet accretion (75). Moreover, miR-146b expression was increased in high fat (HF) diet-induced obese mice, $\mathrm{db} / \mathrm{db}$ mice and ob/ob mice its and expression of its target gene SIRT1 (sirtuin 1) was found to be decreased in white adipose tissue representing induced adipogenesis based on SIRT1 pathway. In addition, in vivo neutralization of miR-146a revealed increased SIRT1 expression relieving the impact of diet-induced obesity (76).

Similarity of rodent model with human NAFLD or NASH is assessed by liver histology, where degree, phenotype of inflammation and fibrosis are rated (17). For example, HF diet leads to the development of the steatotic phenotype that most characterizes human NAFLD/ NASH at early stage. Teufel et al. analysed systemic gene expression profile of liver tissues from patients at different stages of NAFLD compared to various rodent models of NAFLD. Mice model based on high-fat diet seems to be the most similar to human liver affected by NAFLD as documented by resemblance to expression patterns analysed at pathway level (17). Even though the nutritional manipulation-based mouse model is considered the best in terms of correlation to human pathology, no single model is able to reflects the whole process of human disease. However, currently, there are only few models that can mimic the individual aspects of NAFLD (high-fat/high-fructose diet mouse model) $(17,77,78)$.

\section{CONCLUSIONS}

Genetics, environment and dietetic habits play a key role in NAFLD development and progression. Epigenetic factors influence genetic base of the disease and increase individual susceptibility to NAFLD and may stand for great phenotype variability.

Since visceral adipose tissue secretes significant amount of cytokines and adipokines, adipocytes play an important role in fatty liver development. Genes encoding these inflammatory molecules were found to be regulated by epigenetic factors, thus indicating that understanding of differential expression of miRNAs in the adipose tissue is useful for better understanding of mechanisms participating in the development of NAFLD and its progression to advanced stages. Hopefully, circulating miRNAs secreted from tissues, may become promising tool for non-invasive diagnostic differentiation between NASH and NAFLD.

\section{AUTHOR CONTRIBUTIONS}

All authors took part in writing the manuscript. All authors read and approved the final draft.

\section{CONFLICTS OF INTEREST}

The authors declare no conflict of interest. 
ACKNOWLEDGEMENTS

This work was supported by research programs PROGRES Q40/02 and PROGRES Q40/11.

\section{REFERENCES}

1. Eslam M, Sanyal AJ, George J, Panel IC. MAFLD: A Consensus-Driven Proposed Nomenclature for Metabolic Associated Fatty Liver Disease. Gastroenterology 2020; 158(7): 1999-2014.e1.

2. Younossi Z, Anstee QM, Marietti M, et al. Global burden of NAFLD and NASH: trends, predictions, risk factors and prevention. Nat Rev Gastroenterol Hepatol 2018; 15(1): 11-20.

3. Younossi ZM, Koenig AB, Abdelatif D, et al. Global epidemiology of nonalcoholic fatty liver disease - Meta-analytic assessment of prevalence, incidence, and outcomes. Hepatology 2016; 64(1): 73-84.

4. Iravani F, Hosseini N, Mojarrad M. Role of MicroRNAs in Pathophysiology of Non-alcoholic Fatty Liver Disease and Non-alcoholic Steatohepatitis. Middle East J Dig Dis 2018; 10(4): 213-9.

5. Yoo W, Gjuka D, Stevenson HL, et al. Fatty acids in non-alcoholic steatohepatitis: Focus on pentadecanoic acid. PLoS One 2017; 12(12): e0189965.

6. Gurtan AM, Sharp PA. The role of miRNAs in regulating gene expression networks. J Mol Biol 2013; 425(19): 3582-600.

7. Esteller M. Non-coding RNAs in human disease. Nat Rev Genet 2011; 12(12): 861-74.

8. Bartel DP. MicroRNAs: genomics, biogenesis, mechanism, and function. Cell 2004; 116(2): 281-97.

9. Baffy G. MicroRNAs in Nonalcoholic Fatty Liver Disease. J Clin Med 2015; 4(12): 1977-88.

10. Alvarez-Garcia I, Miska EA. MicroRNA functions in animal development and human disease. Development 2005; 132(21): 4653-62.

11. Thomson DW, Bracken CP, Goodall GJ. Experimental strategies for microRNA target identification. Nucleic Acids Res 2011; 39(16): 6845-53.

12. Ma L, Qu L. The function of microRNAs in renal development and pathophysiology. J Genet Genomics 2013; 40(4): 143-52.

13. Deiuliis JA. MicroRNAs as regulators of metabolic disease: pathophysiologic significance and emerging role as biomarkers and therapeutics. Int J Obes (Lond) 2016; 40(1): 88-101.

14. Heneghan HM, Miller N, Kerin MJ. Role of microRNAs in obesity and the metabolic syndrome. Obes Rev 2010; 11(5): 354-61.

15. Uygun A, Ozturk K, Demirci H, et al. The association of nonalcoholic fatty liver disease with genetic polymorphisms: a multicenter study. Eur J Gastroenterol Hepatol 2017; 29(4): 441-7.

16. Asrih M, Jornayvaz FR. Metabolic syndrome and nonalcoholic fatty liver disease: Is insulin resistance the link? Mol Cell Endocrinol 2015; 418(Pt 1): 55-65.

17. Teufel A,Itzel T,ErhartW, etal.Comparison of Gene Expression Patterns Between Mouse Models of Nonalcoholic Fatty Liver Disease and Liver Tissues From Patients. Gastroenterology 2016; 151(3): 513-25.e0.

18. Kassi E, Pervanidou P, Kaltsas G, Chrousos G. Metabolic syndrome: definitions and controversies. BMC Med 2011; 9: 48.

19. Chalasani N, Younossi Z, Lavine JE, et al. The diagnosis and management of non-alcoholic fatty liver disease: practice Guideline by the American Association for the Study of Liver Diseases, American College of Gastroenterology, and the American Gastroenterological Association. Hepatology 2012; 55(6): 2005-23.

20. Nascimbeni F, Pais R, Bellentani S, et al. From NAFLD in clinical practice to answers from guidelines. J Hepatol 2013; 59(4): 859-71.

21. Yki-Järvinen H. Non-alcoholic fatty liver disease as a cause and a consequence of metabolic syndrome. Lancet Diabetes Endocrinol 2014; 2(11): 901-10.

22. Younossi ZM, Golabi P, de Avila L, et al. The global epidemiology of NAFLD and NASH in patients with type 2 diabetes: A systematic review and meta-analysis. J Hepatol 2019; 71(4): 793-801.

23. Rinella ME. Nonalcoholic fatty liver disease: a systematic review. JAMA 2015; 313(22): 2263-73.

24. Wong RJ, Aguilar M, Cheung R, et al. Nonalcoholic steatohepatitis is the second leading etiology of liver disease among adults awaiting liver transplantation in the United States. Gastroenterology 2015; 148(3): 547-55.

25. Sharma H, Estep M, Birerdinc A, et al. Expression of genes for microRNA-processing enzymes is altered in advanced non-alcoholic fatty liver disease. J Gastroenterol Hepatol 2013; 28(8): 1410-5.

26. Klöting N, Berthold S, Kovacs P, et al. MicroRNA expression in human omental and subcutaneous adipose tissue. PLoS One 2009; 4(3): e4699.
27. Gerhard GS, Styer AM, Strodel WE, et al. Gene expression profiling in subcutaneous, visceral and epigastric adipose tissues of patients with extreme obesity. Int J Obes (Lond) 2014; 38(3): 371-8.

28. Capobianco V, Nardelli C, Ferrigno M, et al. miRNA and protein expression profiles of visceral adipose tissue reveal miR-141/YWHAG and miR-520e/RAB11A as two potential miRNA/protein target pairs associated with severe obesity. J Proteome Res 2012; 11(6): 3358-69.

29. Pérez-Pérez R, Ortega-Delgado FJ, García-Santos E, et al. Differential proteomics of omental and subcutaneous adipose tissue reflects their unalike biochemical and metabolic properties. J Proteome Res 2009; 8(4): 1682-93.

30. Kershaw EE, Flier JS. Adipose tissue as an endocrine organ. J Clin Endocrinol Metab 2004; 89(6): 2548-56.

31. Michalakis K, Mintziori G, Kaprara A, Tarlatzis BC, Goulis DG. The complex interaction between obesity, metabolic syndrome and reproductive axis: a narrative review. Metabolism 2013; 62(4): 457-78.

32. Chen $\mathrm{CH}$, Huang $\mathrm{MH}$, Yang JC, et al. Prevalence and risk factors of nonalcoholic fatty liver disease in an adult population of taiwan: metabolic significance of nonalcoholic fatty liver disease in nonobese adults. J Clin Gastroenterol 2006; 40(8): 745-52.

33. Das K, Mukherjee PS, Ghosh A, et al. Nonobese population in a developing country has a high prevalence of nonalcoholic fatty liver and significant liver disease. Hepatology 2010; 51(5): 1593-602.

34. Yu AH, Duan-Mu YY, Zhang Y, et al. Correlation between Non-Alcoholic Fatty Liver Disease and Visceral Adipose Tissue in Non-Obese Chinese Adults: A CT Evaluation. Korean J Radiol 2018; 19(5): 923-9.

35. Chen B, Lam KS, Wang Y, et al. Hypoxia dysregulates the production of adiponectin and plasminogen activator inhibitor-1 independent of reactive oxygen species in adipocytes. Biochem Biophys Res Commun 2006; 341(2): 549-56.

36. Neels JG, Olefsky JM. Inflamed fat: what starts the fire? J Clin Invest 2006; $116(1)$ : 33-5.

37. Christiansen T, Richelsen B, Bruun JM. Monocyte chemoattractant protein-1 is produced in isolated adipocytes, associated with adiposity and reduced after weight loss in morbid obese subjects. Int $\mathrm{J}$ Obes (Lond) 2005; 29(1): 146-50.

38. Weisberg SP, Hunter D, Huber R, et al. CCR2 modulates inflammatory and metabolic effects of high-fat feeding. J Clin Invest 2006; 116(1): $115-24$.

39. Vanni E, Bugianesi E, Kotronen A, et al. From the metabolic syndrome to NAFLD or vice versa? Dig Liver Dis 2010; 42(5): 320-30.

40. Lee YH, Pratley RE. The evolving role of inflammation in obesity and the metabolic syndrome. Curr Diab Rep 2005; 5(1): 70-5.

41. Estep JM, Baranova A, Hossain N, et al. Expression of cytokine signaling genes in morbidly obese patients with non-alcoholic steatohepatitis and hepatic fibrosis. Obes Surg 2009; 19(5): 617-24.

42. Tiniakos DG, Vos MB, Brunt EM. Nonalcoholic fatty liver disease: pathology and pathogenesis. Annu Rev Pathol 2010; 5: 145-71.

43. Xu A, Wang Y, Keshaw H, Xu LY, Lam KS, Cooper GJ. The fat-derived hormone adiponectin alleviates alcoholic and nonalcoholic fatty liver diseases in mice. J Clin Invest 2003; 112(1): 91-100.

44. Linder K, Arner P, Flores-Morales A, Tollet-Egnell P, Norstedt G. Differentially expressed genes in visceral or subcutaneous adipose tissue of obese men and women. J Lipid Res 2004; 45(1): 148-54.

45. van Beek EA, Bakker AH, Kruyt PM, Hofker MH, Saris WH, Keijer $\mathrm{J}$. Intra- and interindividual variation in gene expression in human adipose tissue. Pflugers Arch 2007; 453(6): 851-61.

46. Zhang T, Zhao X, Steer CJ, Yan G, Song G. A negative feedback loop between microRNA-378 and Nrf1 promotes the development of hepatosteatosis in mice treated with a high fat diet. Metabolism 2018; 85: 183-91.

47. Ibrahim MM. Subcutaneous and visceral adipose tissue: structural and functional differences. Obes Rev 2010; 11(1): 11-8.

48. Arner P, Kulyté A. MicroRNA regulatory networks in human adipose tissue and obesity. Nat Rev Endocrinol 2015; 11(5): 276-88.

49. Estep M, Armistead D, Hossain N, et al. Differential expression of miRNAs in the visceral adipose tissue of patients with non-alcoholic fatty liver disease. Aliment Pharmacol Ther 2010; 32(3): 487-97.

50. Celikbilek M, Baskol M, Taheri S, et al. Circulating microRNAs in patients with non-alcoholic fatty liver disease. World J Hepatol 2014; 6(8): 613-20.

51. Cheung O, Puri P, Eicken C, et al. Nonalcoholic steatohepatitis is associated with altered hepatic MicroRNA expression. Hepatology 2008; 48(6): 1810-20.

52. Chartoumpekis DV, Zaravinos A, Ziros PG, et al. Differential expression of microRNAs in adipose tissue after long-term high-fat diet-induced obesity in mice. PLoS One 2012; 7(4): e34872.

53. Latorre J, Moreno-Navarrete JM, Mercader JM, et al. Decreased lipid metabolism but increased FA biosynthesis are coupled with changes 
in liver microRNAs in obese subjects with NAFLD. Int J Obes (Lond) 2017; 41(4): 620-30.

54. Balasubramanyam M, Aravind S, Gokulakrishnan K, et al. Impaired miR-146a expression links subclinical inflammation and insulin resistance in Type 2 diabetes. Mol Cell Biochem 2011; 351(1-2): 197-205.

55. Spengler EK, Loomba R. Recommendations for Diagnosis, Referral for Liver Biopsy, and Treatment of Nonalcoholic Fatty Liver Disease and Nonalcoholic Steatohepatitis. Mayo Clin Proc 2015; 90(9): 1233-46.

56. Dongiovanni P, Meroni M, Longo M, Fargion S, Fracanzani AL. miRNA Signature in NAFLD: A Turning Point for a Non-Invasive Diagnosis. Int J Mol Sci 2018; 19(12): 3966.

57. Pirola CJ, Fernández Gianotti T, Castaño GO, et al. Circulating microRNA signature in non-alcoholic fatty liver disease: from serum non-coding RNAs to liver histology and disease pathogenesis. Gut. 2015;64(5):800-12.

58. Enache LS, Enache EL, Ramière C, et al. Circulating RNA molecules as biomarkers in liver disease. Int J Mol Sci 2014; 15(10): 17644-66.

59. Guduric-Fuchs J, O'Connor A, Camp B, O'Neill CL, Medina RJ, Simpson DA. Selective extracellular vesicle-mediated export of an overlapping set of microRNAs from multiple cell types. BMC Genomics 2012;13:357.

60. Alexander R, Lodish H, Sun L. MicroRNAs in adipogenesis and as therapeutic targets for obesity. Expert Opin Ther Targets 2011; 15(5): 623-36.

61. Poy MN, Eliasson L, Krutzfeldt J, et al. A pancreatic islet-specific microRNA regulates insulin secretion. Nature 2004; 432(7014): 226-30.

62. Tay YM, Tam WL, Ang YS, et al. MicroRNA-134 modulates the differentiation of mouse embryonic stem cells, where it causes post-transcriptional attenuation of Nanog and LRH1. Stem Cells 2008; 26(1): 17-29.

63. Esau C, Kang X, Peralta E, Hanson E, Marcusson EG, Ravichandran LV, et al. MicroRNA-143 regulates adipocyte differentiation. J Biol Chem 2004; 279(50): 52361-5.

64. Bengestrate L, Virtue S, Campbell M, et al. Genome-wide profiling of microRNAs in adipose mesenchymal stem cell differentiation and mouse models of obesity. PLoS One 2011; 6(6): e21305.

65. Kim YJ, Hwang SH, Cho HH, Shin KK, Bae YC, Jung JS. MicroRNA 21 regulates the proliferation of human adipose tissue-derived mesenchymal stem cells and high-fat diet-induced obesity alters microRNA
21 expression in white adipose tissues. J Cell Physiol 2012; 227(1): 183-93.

66. Li M, Liu Z, Zhang Z, Liu G, Sun S, Sun C. miR-103 promotes 3T3-L1 cell adipogenesis through AKT/mTOR signal pathway with its target being MEF2D. Biol Chem 2015; 396(3): 235-44.

67. Can U, Buyukinan M, Yerlikaya FH. The investigation of circulating microRNAs associated with lipid metabolism in childhood obesity. Pediatr Obes 2016; 11(3): 228-34.

68. Ling HY, Ou HS, Feng SD, et al. CHANGES IN microRNA (miR) profile and effects of miR-320 in insulin-resistant 3T3-L1 adipocytes. Clin Exp Pharmacol Physiol 2009; 36(9): e32-9.

69. Lin Q, Gao Z, Alarcon RM, Ye J, Yun Z. A role of miR-27 in the regulation of adipogenesis. FEBS J 2009; 276(8): 2348-58.

70. Grygiel-Górniak B. Peroxisome proliferator-activated receptors and their ligands: nutritional and clinical implications - a review. Nutr J 2014; 13: 17.

71. Pawlak M, Lefebvre P, Staels B. Molecular mechanism of PPARa action and its impact on lipid metabolism, inflammation and fibrosis in non-alcoholic fatty liver disease. J Hepatol 2015; 62 (3): 720-33.

72. Sugden MC, Caton PW, Holness MJ. PPAR control: it's SIRTainly as easy as PGC. J Endocrinol 2010; 204(2): 93-104.

73. Zhong X, Liu H. Honokiol attenuates diet-induced non-alcoholic steatohepatitis by regulating macrophage polarization through activating peroxisome proliferator-activated receptor $\gamma$. J Gastroenterol Hepatol 2018; 33(2): 524-32.

74. Xie H, Lim B, Lodish HF. MicroRNAs induced during adipogenesis that accelerate fat cell development are downregulated in obesity. Diabetes 2009; 58(5): 1050-7.

75. Price NL, Holtrup B, Kwei SL, et al. SREBP-1c/MicroRNA 33b Genomic Loci Control Adipocyte Differentiation. Mol Cell Biol 2016; 36(7): 1180-93.

76. Ahn J, Lee H, Jung CH, Jeon TI, Ha TY. MicroRNA-146b promotes adipogenesis by suppressing the SIRT1-FOXO1 cascade. EMBO Mol Med 2013; 5(10): 1602-12.

77. Wong VW, Wong GL, Choi PC, et al. Disease progression of non-alcoholic fatty liver disease: a prospective study with paired liver biopsies at 3 years. Gut 2010; 59(7): 969-74.

78. Imajo K, Yoneda M, Kessoku T, et al. Rodent models of nonalcoholic fatty liver disease/nonalcoholic steatohepatitis. Int J Mol Sci 2013; 14(11): 21833-57. 